\title{
Effect of Extracorporeal Life Support on Survival When Applied to All Patients With Congenital Diaphragmatic Hernia
}

\author{
By Cynthia N. Steimle, Funda Meric, Ronald B. Hirschl, Mary Bozynski, Arnold G. Coran, and Robert H. Bartlett \\ Ann Arbor, Michigan
}

\begin{abstract}
- Extracorporeal life support (ECLS) has been used for neonates with congenital diaphragmatic hernia (CDH) and respiratory failure at the authors' hospital since June 1981. In 1988 , criteria for inclusion in ECLS were broadened to include "nonhoneymoon" infants (honeymoon: best postductal $\mathrm{PaO}_{2}$ of $>50 \mathrm{~mm} \mathrm{Hg}$ ). To evaluate the impact of this approach on the treatment of $\mathrm{CDH}$, the authors reviewed the records of all newborns managed at their institution, since the availability of ECLS in 1981, who were symptomatic with CDH in the first 24 hours of life $(n=111)$. The patients were divided chronologically into two groups: 1981 to 1987 (early ECLS, $n=36$ ) and 1988 to 1993 (expanded ECLS, $n=75$ ). The data demonstrate that the number of CDH patients managed at our institution each year has increased (1981 to $1987=6,1988$ to $1993=14$ ) as has the severity of associated respiratory insufficiency ( $\%$ of patients with best $\mathrm{PaO}_{2}$ of $\leq 50 \mathrm{~mm} \mathrm{Hg}$ : 1981 to $1987=6 \%, 1988$ to $1993=28 \%$ ). Overall, the survival rate was lower for patients in the expanded ECLS group $(59 \% \vee 75 \% ; P=.121)$. When the survival rates for patients supported with ECLS postoperatively were compared for the expanded and early groups, a significant difference $159 \% v$ $80 \% ; P<.05)$ was noted. However, when ECLS patients with a best $\mathrm{PaO}_{2}$ of $\leq 50 \mathrm{~mm} \mathrm{Hg}$ were excluded from this analysis, there was no significant difference between the two groups $(74 \% \vee 80 \%)$, indicating that the inclusion of nonhoneymoon patients as potential ECLS candidates has accounted for a significant portion of the observed decrease in survival among CDH patients managed with ECLS. The survival rate of salvageable CDH patients with a best $\mathrm{PaO}_{2}$ of $\leq 50 \mathrm{~mm} \mathrm{Hg}$ increased slightly for the expanded ECLS group, to $27 \%$ (4 of $15)$, versus $0 \%$ ( 0 of 2 ) for the early ECLS group. The authors conclude that a change in the population of $\mathrm{CDH}$ patients presenting to their institution along with application of ECLS to CDH patients without a honeymoon has resulted in a reduction in the survival rate, but has allowed only a modest $27 \%$ survival rate for patients who were previously considered nonsalvageable.
\end{abstract}

Copyright 1994 by W.B. Saunders Company

INDEX WORDS: Diaphragmatic hernia, congenital, respiratory failure, extracorporeal support.

I N 1988, OUR GROUP reported an increase in the overall survival rate for infants with congenital diaphragmatic hernia (CDH), from $50 \%$ to $76 \%$, through the application of extracorporeal life support (ECLS) in the setting of severe respiratory failure. ${ }^{1}$ At the time of our previous report, we commonly excluded patients from consideration for ECLS if they had never had a best postductal $\mathrm{PaO}_{2}$ of more than $50 \mathrm{~mm} \mathrm{Hg}$. It was believed that these patients had severe pulmonary hypoplasia, which would preclude recovery despite prolonged courses of ECLS. However, subsequent studies suggested that such criteria were nonspecific in predicting mortality and not appropriate for use as ECLS exclusion criteria. ${ }^{2}$ Therefore, in 1988, all patients with $\mathrm{CDH}$ who had persistent respiratory failure despite maximal conventional support were considered candidates for ECLS, provided their weight was more than $2 \mathrm{~kg}$, their gestational age was more than 34 weeks, and they had no severe genetic anomalies or other abnormalities that would preclude viable existence. Herein we summarize our experience over the last 12 years, since ECLS has been available, and analyze the effect of such changes in ECLS selection criteria.

\section{MATERIALS AND METHODS}

We performed a retrospective review of the records of all patients with $\mathrm{CDH}$ treated at the University of Michigan Medical Center (UMMC), C.S. Mott Children's Hospital, from June 1981 to June 1993.

Before 1981, ECLS therapy was not available at our center for the management of $\mathrm{CDH}$ patients with severe pulmonary failure. Subsequent to 1981 , all patients who had significant respiratory failure despite maximal therapy with conventional ventilatory and pharmacological support were considered for ECLS. Criteria that, on retrospective review, could reliably identify infants with projected mortality rates of $80 \%$ to $100 \%$ with conventional ventilatory support were used to select patients for treatment with ECLS. ${ }^{3.4}$ The inclusion criteria include (1) acute deterioration, with $\mathrm{PaO}_{2}$ of less than $40 \mathrm{~mm} \mathrm{Hg}$ or $\mathrm{pH}$ of less than 7.15 for more than 2 hours: (2) oxygen index (OI) of more than 40 on three of five measurements between 30 and 60 minutes apart (where $\mathrm{OI}=\mathrm{FiO}_{2} \times$ mean airway pressure $\times 100$, divided by postductal $\mathrm{PaO}_{2}$ ); or (3) severe barotrauma. Previously reported exclusion criteria were developed to eliminate patients from consideration for ECLS if reasonable expectation of meaningful survival could not be achieved. ${ }^{1}$ The exclusion criteria were (1) age of more than 10 days; (2) gestational age of less than 35 weeks; (3) weight of less than $2 \mathrm{~kg}$; (4) preexisting severe intracranial hemorrhage; (5) serious coexistent genetic anomaly; (6) uncorrectable cyanotic heart disease; (7) moribund condition; or (8) ongoing cardiopulmonary resuscitation before cannulation was begun. In our initial experience, patients were excluded from consideration for ECLS if they never had a postductal $\mathrm{PaO}_{2}$ of more than $50 \mathrm{~mm} \mathrm{Hg}$ (ie. if they never had "honeymoon" period). This exclusion criterion was applied to identify "unsalvageable" patients with bilateral pulmonary hypopla-

From the Departments of Surgery and Pediatrics. The University of Michigan Medical Center, Ann Arbor, MI.

Presented at the 1993 Annual Meeting of the Section on Surgery of the American Academy of Pediatrics, Washington, DC, October 29-31, 1993.

Address reprint requests to Ronald B. Hirschl. MD, L2110 Maternal Child Health Care Center, 1500 E Medical Center Dr, Ann Arbor, MI 48109-0245.

Copyright 1994 by W.B. Saunders Company

0022-3468/94/2908-0009\$03.0010 
sia. However, beginning in 1988, this exclusion criterion was no longer applied, and ECLS was then used in nonhoneymoon patients. Therefore, two eras, or groups, each with individual management strategies for $\mathrm{CDH}$, were identified at our institution. Group 1 patients were treated from June 1981 to December 1987 ("early ECLS era"). Group 2 patients were treated from January 1988 to June 1993 ("expanded ECLS era").

An additional difference in management strategy between the early and expanded ECLS groups lies in the timing of operative repair. Before 1988, all patients were operated on as soon as it was feasible; ECLS was used only in the postoperative period. When we expanded our criteria for ECLS, it was noted that newborns with severe respiratory insufficiency would not be able to tolerate surgery unless bypass was initiated preoperatively. Therefore, we began to institute ECLS in these neonates emergently, and the operation was performed while they were on ECLS support. More recently, in 1993, we began to attempt to wean these patients from ECLS support before operative repair. Therefore, all ECLS patients in group 1 had repair before ECLS, but patients in group 2 could be repaired pre-ECLS, on ECLS, or post-ECLS.

The two groups of patients were assessed for differences in severity of illness and differences in survival rates. Data were compared using Fisher's exact test and $\chi^{2}$ analysis.

\section{RESULTS}

Between June 1981 and June 1993, 111 patients with $\mathrm{CDH}$ diagnosed in the first 24 hours of life were treated at the University of Michigan, C.S. Mott Children's Hospital. Thirty-six of them were treated from June 1981 to December 1987 (early ECLS era; group 1), and 75 were treated from January 1988 to June 1993 (expanded ECLS era; group 2). Therefore, the number of $\mathrm{CDH}$ patients managed each year at our institution increased from 6 per year (1981 to 1987 ) to 14 per year (1988 to 1993) over the study period.

Twenty-six of the 36 patients (72\%) in the early ECLS group were born at outlying hospitals; seven of whom $(27 \%)$ underwent operative repair before being transferred to our facility. In the current expanded ECLS era, 52 of $75(70 \%)$ were born at outlying hospitals, 16 of whom $(31 \%)$ had repair before transport.

Between the time periods, there was a substantial increase in the proportion of patients for whom the diagnosis was made in utero. In the early ECLS era, eight patients were identified as having $\mathrm{CDH}$ based on prenatal ultrasound examination $(8$ of $36 ; 22 \%)$; seven of these patients were delivered at our institution. In group 2, 30 patients had the diagnosis made in utero ( 30 of $75 ; 40 \%) ; 20$ of them were delivered at our institution. Another 14 patients in this group had undergone prenatal ultrasound examination, and $\mathrm{CDH}$ had not been detected.

As seen in Table 1, there were no significant differences between the two groups in terms of gestational age or birth weight. There was also no significant difference in the percentage of patients
Table 1. Patient Characteristics

\begin{tabular}{lccc}
\hline \multicolumn{1}{c}{ Characteriatic } & Early ECLS & Expanded ECLS & $P$ \\
\hline No. of patients & 36 & 75 & \\
Gestational age $(w \mathrm{k})$ & $38.3 \pm 2.8$ & $38.0 \pm 2.9$ & NS \\
Birth weight $(\mathrm{kg})$ & $3.11 \pm 0.62$ & $2.92 \pm 0.69$ & NS \\
Associated anomalioes & $22 \%$ & $27 \%$ & NS \\
Left-sided hernia & $78 \%$ & $81 \%$ & NS \\
Primary repair & $84 \%$ & $58 \%$ & NS \\
Born at UMMC & $28 \%$ & $30 \%$ & NS \\
Repair at UMMC & $73 \%$ & $69 \%$ & NS \\
Diagnosis in utero & $22 \%$ & $40 \%$ & $<.01$ \\
Required ECLS & $42 \%$ & $65 \%$ & $<.05$ \\
PaO ${ }_{2} \leq 50$ mm Hg & $6 \%$ & $28 \%$ & $<.05$ \\
\hline
\end{tabular}

with associated anomalies or in the percentage who had a left-sided hernia. The proportion of patients who met criteria for and in whom ECLS was used increased with time, as did the proportion of $\mathrm{CDH}$ patients who required a muscle flap or prosthetic reconstruction of the diaphragm at the time of $\mathrm{CDH}$ repair. In addition, the proportion of patients with a best $\mathrm{PaO}_{2}$ of $\leq 50 \mathrm{~mm} \mathrm{Hg}$ increased substantially $(6 \%$ for the early ECLS group, $28 \%$ for the expanded ECLS group).

The survival rates appear in Table 2 . In the early ECLS group, overall survival was 27 of $36(75 \%)$. Of the 27,20 were treated postoperatively with conventional ventilation alone, and 15 survived (75\%). In addition, 12 of 15 patients treated postoperatively with ECLS survived (80\%). Of the six patients who died without the use of ECLS, two were excluded because their best $\mathrm{PaO}_{2}$ did not exceed $50 \mathrm{~mm} \mathrm{Hg}$, two bccause of genetic anomalies, and two because of prematurity.

In the expanded ECLS group, overall survival was 44 of $75(59 \%)$. Seventeen of the 44 were treated with conventional ventilation alone, and 15 survived ( $88 \%)$. The total number of patients treated with ECLS was 49; of these, 29 survived (59\%). These survival rates are lower than those for the early ECLS group; however, the difference was not significant (Fig 1). Twenty-nine patients had repair before initiation of ECLS, with 21 survivors (72\%). Seventeen patients had repair while on ECLS, with six survivors (35\%).

Table 2. Survival Data

\begin{tabular}{lccc}
\hline \multicolumn{1}{c}{ Group } & Early ECLS & Expanded ECLS & $P$ \\
\hline All patients & $36(75)$ & $75(59)$ & $\mathrm{NS}$ \\
All potential ECLS patients & $32(84)$ & $65(67)$ & $\mathrm{NS}$ \\
$\mathrm{PaO}_{2}>50 \mathrm{~mm} \mathrm{Hg}$, viable & $30(90)$ & $50(80)$ & $\mathrm{NS}$ \\
$\mathrm{PaO}_{2} \leq 50 \mathrm{~mm} \mathrm{Hg}$, viable & $2(0)$ & $15(27)$ & $\mathrm{NS}$ \\
$\mathrm{ECLS}, \mathrm{PaO}_{2} \geq 50 \mathrm{~mm} \mathrm{Hg}$ & $15(80)$ & $34(74)$ & $\mathrm{NS}$ \\
All ECLS-treated patients & $15(80)$ & $49(59)$ & $<.05$ \\
\hline
\end{tabular}

NOTE. Numbers in parentheses are percentages. 


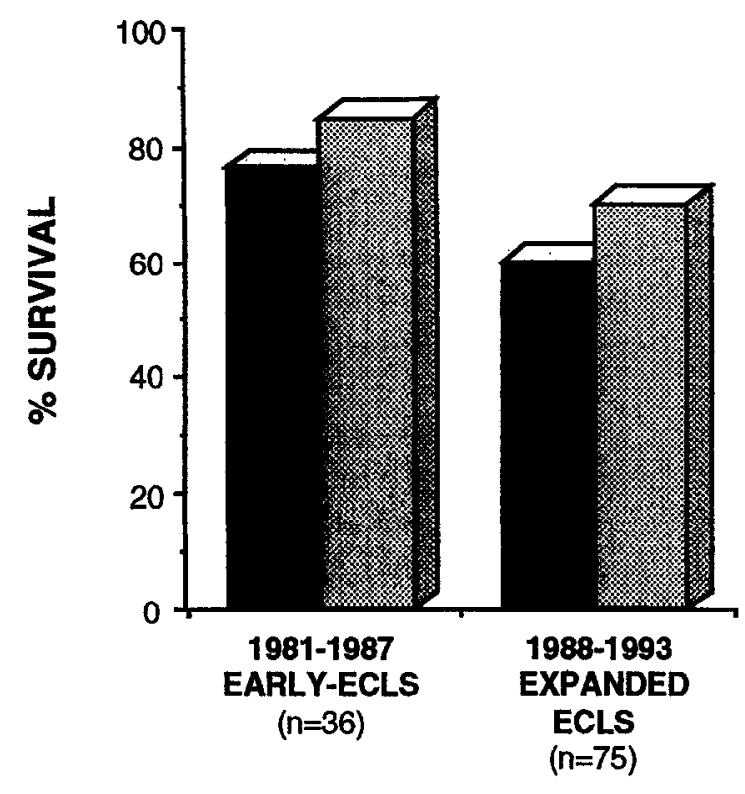

Fig 1. Survival rates for patients with $\mathrm{CDH}$. No statistically significant differences are seen between each era. "Viable" patients ishaded bars) are those who met inclusion criteria for ECLS. Solid bars denote all patients.

Three patients were weaned from ECLS before CDH repair was attempted, with two survivors $(67 \%)$.

The extension of ECLS to patients with more severe respiratory failure clearly diminished survival in the group treated with ECLS. In the expanded ECLS group, there were 21 patients with a best postductal $\mathrm{PaO}_{2}$ of $\leq 50$. However, only 15 were placed on ECLS; the remainder were excluded by other criteria. Four of these survived $(27 \%)$. These patients would have been excluded from ECLS under our previous criteria. There was a significant difference in the survival rates between patients managed with ECLS in the expanded era and those managed in the early era $(59 \% \vee 80 \% ; P<.05)$. However, when only the patients whose best $\mathrm{PaO}_{2}$ exceeded $50 \mathrm{~mm}$ $\mathrm{Hg}$ (managed with ECLS) were considered, there was no significant difference between the survival rates (80\% for the early group, $74 \%$ for the expanded group). The period of time on ECLS for patients whose best $\mathrm{PaO}_{2}$ was $\leq 50 \mathrm{~mm} \mathrm{Hg}$ was $14.5 \pm 7.1$ days; this is significantly different from that of patients with a best $\mathrm{PaO}_{2}$ of more than $50 \mathrm{~mm} \mathrm{Hg}(9.9 \pm 6.5$ days; $P<.05)$. The average hospital stay for the four survivors whose best $\mathrm{PaO}_{2}$ was $\leq 50 \mathrm{~mm} \mathrm{Hg}$ was 2 months (range, 31 to 99 days). The mean period of hospitalization did not differ significantly between these patients and those whose best $\mathrm{PaO}_{2}$ exceeded 50 $\mathrm{mm} \mathrm{Hg} \mathrm{(52} \pm 31$ days $v 49 \pm 51$ days). Follow-up of these four survivors (at 18 to 30 months) showed that one died at home of unknown causes a few months after hospital discharge. One required a fundoplica- tion for gastroesophageal reflux (GER), but is otherwise well. One child is slightly delayed neurologically and has reactive airway disease. This patient also had findings of GER and underwent a fundoplication procedure. The fourth child is normal. None of the three surviving patients currently requires supplemental oxygen.

It is interesting to note that the patients for whom $\mathrm{CDH}$ was diagnosed in utero in the early ECLS era all had a best $\mathrm{PaO}_{2}$ of more than $50 \mathrm{~mm} \mathrm{Hg}$, and their overall survival rate was $75 \%$ (six of eight). However, for the expanded ECLS group, only 17 of $30(57 \%)$ had a best $\mathrm{PaO}_{2}$ of more than $50 \mathrm{~mm} \mathrm{Hg}$, with an overall survival rate of $37 \%$ ( 11 of 30 ).

Seventeen patients had $\mathrm{CDH}$ repair while on ECLS support; there were six survivors (35\%) in the expanded ECLS era. Ten of the 17 had a best $\mathrm{PaO}_{2}$ of $\leq 50 \mathrm{~mm} \mathrm{Hg}$; four of the 10 survived. Figure 2 shows the survival data for the patients repaired before and during ECLS.

The incidence of significant complications during ECLS was low in both eras. Major bleeding was the most common complication, occurring in four patients in the early era (26\%) and four in the expanded era $(8 \%)$. In the expanded era, three children were removed from ECLS because of severe intracranial hemorrhage. A fourth child had severe postoperative bleeding, which required discontinuation of ECLS; that child subsequently died of respiratory insufficiency. There were no other complications of ECLS that directly contributed to patient death. In addition, there were no mechanical complications of ECLS that resulted in patient death.

In the early ECLS era, there were nine deaths. Six of the nine children who died were excluded from ECLS support; two of these were premature, two had genetic anomalies, and two did not have a postductal $\mathrm{PaO}_{2}$ of more than $50 \mathrm{~mm} \mathrm{Hg}$. One child died of intracranial hemorrhage while on ECLS support. The other two deaths occurred late-one secondary to

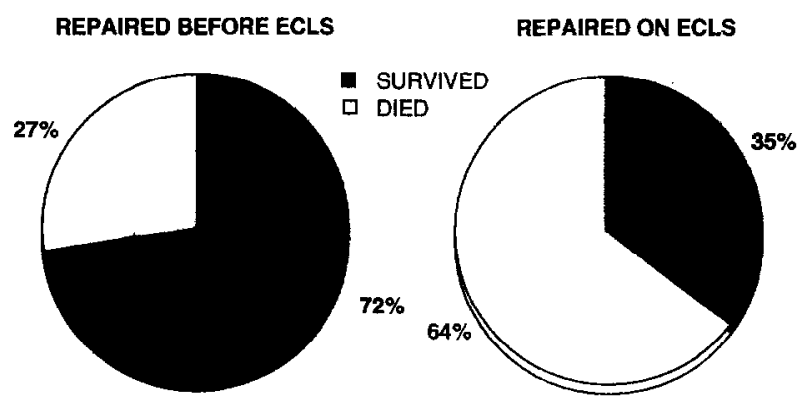

Fig 2. Expanded ECLS (1988-1993). Survival rates for patients treated with ECLS before or during CDH repair. Patients repaired while on ECLS had a significantly lower survival rate than those repaired before ECLS. 
sepsis, and the other because of bronchopulmonary dysplasia (BPD).

In the expanded ECLS era, there were 31 deaths. Ten occurred in patients who were excluded from ECLS support because of prematurity (4), multiple anomalies (3), pre-ECLS cardiac arrest (2), or intraoperative death (1). One death occurred late, in a child who had been transferred to another hospital for care; she died of acute onset of respiratory failure, which was attributed to BPD. The causes of death for the 20 patients managed with ECLS included persistent respiratory failure (7), bleeding complications (5), sequelae of ischemic neurological injury (3), necrotizing enterocolitis (2), and multisystem organ failure (3). All three in the latter group had had renal failure during ECLS. Two had concomitant persistent respiratory failure, but one had been successfully weaned from ECLS and died 12 days later, after the development of sepsis from acalculous cholecystitis.

\section{DISCUSSION}

Reports of survival of $\mathrm{CDH}$ patients published from 1965 to 1985 show mortality rates in the range of $50 \% .{ }^{1}$ The causes of death can be grouped as follows: (1) pulmonary hypoplasia, (2) persistent pulmonary hypertension of the newborn, and (3) associated anomalies. ${ }^{5}$ ECLS was introduced to support the neonate while persistent pulmonary hypertension resolves.

Selection of patients for treatment with ECLS has been a topic of great debate. Multiple criteria have been developed by Bohn et al, Stolar et al, and others, which serve as predictors of high mortality in the $\mathrm{CDH}$ patient managed with conventional mechanical ventilation. ${ }^{6,7}$ However, although these various criteria appear to have relevance in terms of predicting those who will die with conventional support alone, the issue of which patients will succumb even if more aggressive intervention is instituted (such as ECLS) remains unresolved. Attempts to develop criteria that identify patients with inadequate lung parenchyma to support life have generally been unsuccessful. ${ }^{2,8}$ Consequently, most centers have begun to offer ECLS support to any neonate with $\mathrm{CDH}$ for whom conventional mechanical ventilatory support has failed.

In 1988, at our institution, we began to use ECLS support in any neonate for whom conventional mechanical ventilatory support had failed. A review of our survival data since that time, as compared with our previous survival figures, indicates that overall survival has diminished since the change was instituted $(59 \% \vee 75 \%)$, although this difference is not statistically significant. In addition, the survival rate for all patients managed with ECLS decreased significantly between the early and expanded eras, whereas the survival rate for those whose best $\mathrm{PaO}_{2}$ exceeded $50 \mathrm{~mm} \mathrm{Hg}$ has not decrease significantly. These data allow us to conclude that it is the inclusion of these more severely affected newborns that has accounted for the major portion of the observed decrease in survival among paticnts managed with ECLS. Expansion of criteria to include $\mathrm{CDH}$ patients with a best $\mathrm{PaO}_{2}$ of $\leq 50 \mathrm{~mm} \mathrm{Hg}$ has resulted in only a small $(27 \%)$ increase in survival among the patients who were previously considered unsalvagcablc. As is evident from the long courses on ECLS and the prolonged hospital stay for survivors, considerable resources are expended in an attempt to salvage very few patients. We believe that patients with a best $\mathrm{PaO}_{2}$ of $\leq 50 \mathrm{~mm} \mathrm{Hg}$ remain relatively poor candidates for ECLS and that cach centcr should evaluate whether their experience in this high-mortality group warrants the continued inclusion of such patients as ECLS candidates.

The number of cases diagnosed in utero has increased dramatically, from eight $(22 \%)$ in the early ECLS era to $30(44 \%)$ in the expanded era. All eight of the early patients had a best $\mathrm{PaO}_{2}$ of more than 50 $\mathrm{mm} \mathrm{Hg}$; only three required ECLS support, and six of the eight $(75 \%)$ survived. In contrast, in the expanded era, only 17 of $30(57 \%)$ had a best $\mathrm{PaO}_{2}$ of more than $50 \mathrm{~mm} \mathrm{Hg}$, and survival was only 11 of $30(37 \%)$. It is evident that the recent application of in utero ultrasonography has led to an increase in the severity of respiratory insufficiency of $\mathrm{CDH}$ patients managed at our institution. We suggest that this is because of changes in perinatal referral patterns, which have led to more expeditious and efficient transfer, either before or after birth, of the fetus/newborn with known $\mathrm{CDH}$. In utero diagnosis is also responsible for the increase in referrals to our institution (from 36 patients in the early era to 75 in the expanded era).

The timing of surgical repair for infants with $\mathrm{CDH}$ has been a subject of recent debate. Traditionally, emergency surgical repair of $\mathrm{CDH}$ has been performed to reduce the herniated viscera, to eliminate the risk of bowel incarceration, and to eliminate compression of the lungs and resultant poor gas exchange. However, surgical repair of $\mathrm{CDH}$ has been shown to result in a reduction in pulmonary compliance. ${ }^{9}$ In already marginally ventilating infants, this decreased compliance can have severe clinical consequences. Bohn et al suggested that delayed surgical repair might allow reversal of pulmonary artery hypertension and improved survival. ${ }^{6}$ However, some in- 
fants are not able to oxygenate and ventilate adequately at the time of initial presentation. Our current protocol allows placement of such infants on ECLS, with delay in operative repair until the patients can be successfully weaned from ECLS support. Infants who present to our center in stable condition are maintained in the neonatal intensive care unit and are electively repaired before extubation, usually when they are at least 72 hours of age. The effect of this approach on the outcome of newborns with $\mathrm{CDH}$ managed at our institution is yet to be determined.

\section{REFERENCES}

1. Heiss $\mathrm{K}$, Manning $P$, Oldham KT, et al: Reversal of mortality for congenital diaphragmatic hernia with ECMO. Ann Surg 209:225230,1988

2. Newman KD, Anderson KD, VanMeurs K, et al: Extracorporeal membrane oxygenation and congenital diaphragmatic hernia: Should any infant be excluded? J Pediatr Surg 25:1048-1053, 1990

3. Bartlett RH, Toomasian J, Roloff D, et al: Extracorporeal membrane oxygenation (ECMO) in neonatal respiratory failure: 100 cases. Ann Surg 204:236-245, 1986

4. Bartlett RH, Andrews AF, Toomasian JM, et al: Extracorporeal membrane oxygenation for newborn respiratory failure: Fortyfive cases. Surgery 92:425-433, 1982

5. Dibbins AW, Weiner ES: Mortality from neonatal diaphragmatic hernia. J Pediatr Surg 9:653-662, 1974
6. Bohn D, Tamura M, Perrin D, et al: Ventilatory predictors of pulmonary hypoplasia in congenital diaphragmatic hernia, confirmed by morphologic assessment. J Pediatr 111:423-431, 1987

7. Stolar C, Dillon P, Reyes C: Selective use of extracorporeal membrane oxygenation in the management of congenital diaphragmatic hernia. J Pediatr Surg 23:207-211, 1988

8. Bailey PV, Connors RH, Tracy TF, et al: A critical analysis of extracorporeal membrane oxygenation for congenital diaphragmatic hernia. Surgery 106:611-616, 1989

9. Sakai H, Tamura M, Hosokawa V, et al: Effect of surgical repair on respiratory mechanics in congenital diaphragmatic hernia. J Pediatr 111:432-438, 1987

\section{Discussion}

K. Lally (Houston, TX): The authors have nicely shown that if entry criteria for ECMO in patients with diaphragmatic hernia are expanded, survival not unexpectedly decreases. Certainly, increasing the inborn population would give you a high number of these very high-risk patients. In the most recent series of your patients, you had 23 in-born and 52 out-born patients. I'd like to know, did you have any difference in survival between these two groups?

Secondly, almost every predictor of nonsurvival published to date has not truly withstood the test of time, although there is growing consensus that the inability to ventilate by preductal $\mathrm{PCO}_{2}$ may reflect inadequate alveolar surface area and be a reasonable predictor. Did any of your survivors have a best preductal $\mathrm{PCO}_{2}$ of greater than 50 ?

And finally, improvement in survival with ECLS has led to growing recognition of the long-term problems with $\mathrm{CDH}$ survivors, including late deaths from right-sided heart failure, gastroesophageal reflux, and recurrent herniation. Do you have any late follow-up information for your ECLS survivors?

C.N. Steimle (response): We did not see any difference in outcome between the in-born and out-born groups. We believe this is probably because the ability to treat the patients quickly when they are in-born is balanced by the fact that we also do see sicker patients who, had they been out-born, would have never made it to our center for therapy.

We did not routinely measure the best $\mathrm{PCO}_{2}$ until about a year ago. We are now looking at this as one of our predictors of mortality. We're also looking at other indicators of mortality, including left ventricular mass.

In the follow-up of the four surviving patients, who had a best $\mathrm{PaO}_{2}$ of less than 50, there was one late death; however, the other three patients are doing well, are functionally intact, and have no significant problems. 